\title{
Comparison of Recognizing Russian Alphabets in the Associative Random Access Memory (ARAM) and the Classical Memory (RAM)
}

\author{
Md. Abdul Malek, PhD \\ Assistant Professor \\ Department of Computer Science \\ \& Engineering \\ Comilla University \\ Comilla, Bangladesh
}

\author{
Md. Mohibullah \\ Student (M.Sc. - Thesis) \\ Department of Computer Science \\ \& Engineering \\ Comilla University \\ Comilla, Bangladesh
}

\begin{abstract}
In this paper, a comparison between Associative Random Access Memory(ARAM) and classical memory,also known as Random Access Memory (RAM), for recognizing symbols (e.g., letters, words, images, gestures etc.) has been made. To do it, firstly an efficient system to recognize symbols in an associative environment has beeen performed. For instance, they have taken the system to recognize the letters in the Russian Alphabet. This paper represents a method of formation and recognition of codes for the letters in a seven segment elements of matrices in the associative cells of the ARAM. For the recognition of codes of letters in the ARAM, the algorithm is developed throughout the paper and they use the experimental results to illustrate the comparison between ARAM and RAM.
\end{abstract}

\section{Keywords}

Recognition of symbol, associative memory, RAM, algorithm, comparison.

\section{INTRODUCTION}

In this world, most of the works, that are related to the computer memories, are done using the classical memories (RAMs).The performance and speed of basic components of any device related to the classical architecture are in the edge of developing. So it is very cumbersome to increase the performance and speed of these basic components. One possible solution may come out, if any change in whole classical architecture's organization can be made to combine the processing and storing functions. But the problem becomes much more severe. However, one of the most important perspective directions of solutions is the use of ARAM of IBM. Associative Random Access Memory, also known as Content Addressable Memory (CAM), is a special type of computer memory. Generally, it is used in certain applications requiring high speed searching.

Three concepts differs the ARAM from the RAM:

1. Collective access to all data stored in ARAM.

2. In associative memory, storage locations are accessed by their contents of data or the parts of contents, not by the position.

3. ARAM is used for the performance of a variety of logical and mathematical operations i.e., when one selects some data from ARAM to perform logical and mathematical operations, it is easily done without the participation of CPU.

For these reasons, they select their work-the recognition of symbols in the associative environment.

\section{WORKING PRINCIPALS OF ARAM WITH RESPECT TO RUSSAIN'S SYMBOL}

The concept of symbols in matrixes ARAM is based on the following: any symbol of the Russian alphabet is represented with a set of the associated vertical and horizontal pieces of lines and a diagonal direction with a slope $45^{\circ}$ and $135^{\circ}$.

For recognition it is supposed to place the symbol in matrix ARAM by following rules:

1. The image of a symbol begins with an extreme left column (Щ, Ш, Н, Г, Ц, Е, Ы, П, Р, Ч, М, Ь,) and extreme top line $(\Gamma, \Pi, \mathrm{A}, \mathrm{B}, Л, \mathrm{~T}, \mathrm{Б})$ of matrixes ARAM. Symbols Л and Д will not start from the left top element of a matrix (shown in Figure from 1 to 4 ).

2. Across on a horizontal and on a vertical contour symbol uses 1 bit that is, thickness is considered to a contour of a symbol individual.

3. Codes of adjacent horizontal or vertical parallel pieces of symbols (for example, vertical pieces in letters И, Н, Ш, Щ, П, А) settle down accordingly in lines or columns, from each other for one line or one column, that is, between parallel pieces there are codes of space - «Zero» codes.

4. Proceeding from item 1-3, for coding all letters of the Russian alphabet 5 lines of matrix ARAM are required. Quantity of columns is required from 3 for maximum letters and up to 6 for the letter Щ.

5. Diagonal lines of symbols should not have less than three elements of a matrix adjacent on a diagonal with one value. For example, the code of the letter $M$ requires $5 \times 5$ elements of a matrix and a code of a direct diagonal is equal to 11101 , the code of a return diagonal is equal to 10111.

6. At coding vertical pieces of the symbols engaging an incomplete column, e. g., for symbols Б, Ч, $\Phi, \mathrm{P}, \mathrm{Y}, \mathrm{b}$ Ы, Я, necessary three bits, accepting value «1». 
7. Feature of coding of symbols $Э$ and 3 is that codes of middle lines differ one bit: for symbol $Э$ - code 011 , for

symbol 3- code 111.

$5 \times 6$

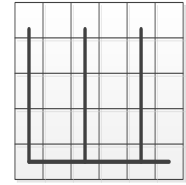

Figure 1

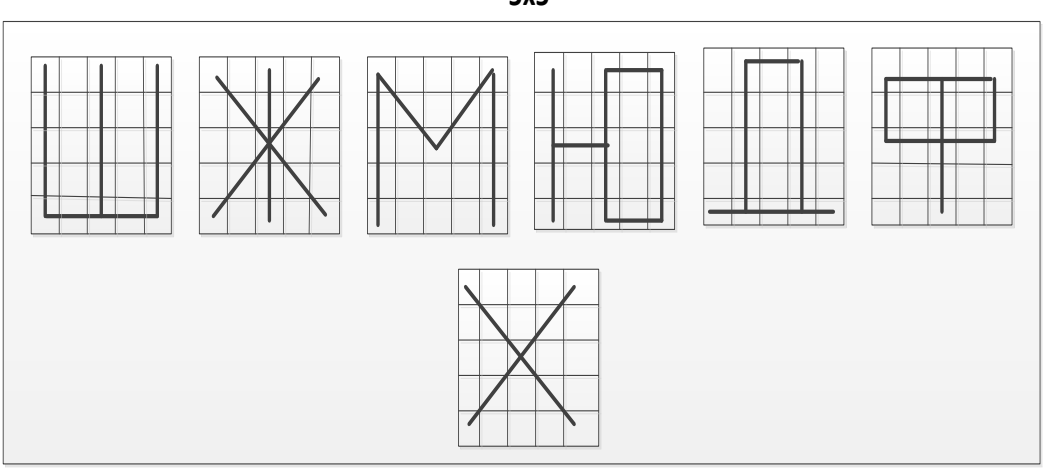

Figure 2
$5 \times 4$

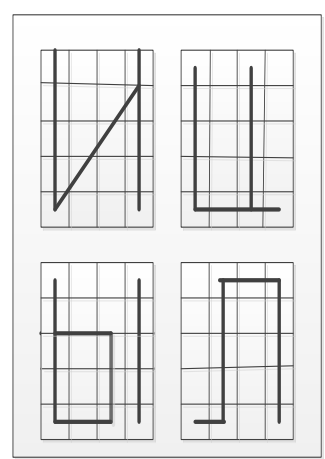

Figure 3
$5 \times 3$

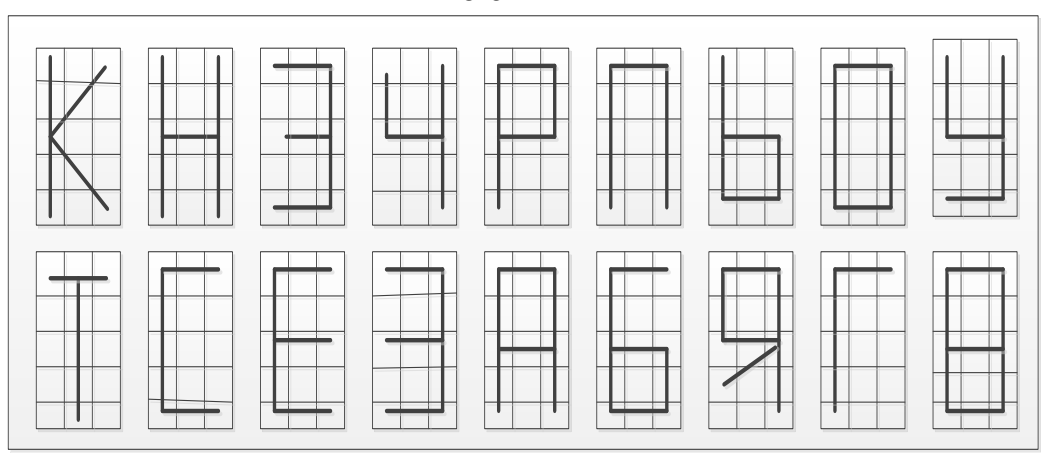

Figure 4

Figure (1-4): Russian Symbols in ARAM'S Matrices

\section{FORMATION'S CODES ALGORITHM}

The algorithm for formation of codes of library of pieces of the symbols which are stored in the associative storage and being in essence by codes of signs of symbols is based on following preconditions:

1. The code of a symbol engages a matrix $5 \times 6$ (5 lines, 6 columns).

2. «1» values of elements of a matrix form shape of a symbol.

The essence of algorithm for formation of codes of the symbols forming library of codes of samples of symbols, consists in the following:

Step 1:

1. On the register of interrogation of the block of register RA [BR1], [1]-[2] and the register of interrogation of the block of register RA [BR2], codes 111111 and 11111 move at the same time.

2. Check of these codes on concurrence to codes accordingly all 5 lines and all 6 columns of letters of the alphabet is carried out and the result is recorded in appropriating categories of registers of fixing RS1 and RS2.
3. Received in RS1 and RS2 the pair codes forms the first code of a sign of the given letter which is recorded in the first section of a matrix of library of samples.

Step 2:

1. Place the code $111 \mathrm{mmm}$ on RA [BR1] and the code $\mathrm{mm} 101$ on RA [BR2], where $\mathrm{m}$ is the disguised category which is not participating in check on concurrence of codes.

2. Fulfilling the check on concurrence of these codes to codes of letters of the alphabet accordingly all 5 lines and all 6 columns of letters of the alphabet and the result is recorded in appropriating categories of registers of fixing RS1 and RS2.

3. Received in RS1 and RS2, the second pair codes form the second code of a sign of the given letter which is recorded in the second section of a matrix of library of samples.

Step 3:

The received two pair's codes are kept in memory of library of codes of samples (signs) of letters. Thus, memory of library of codes will consist of two parts on 11 categories, that is, a full code of signs depending on the letter 11 bits (for letters of M, T, X, Щ) or 22 bits (for the others). 


\section{RECOGNITION'S CODES ALGORITHM}

The algorithm for recognition of codes of letters in the associative environment [3] consists in the following:

1. Place the code of the unknown letter in memory ARAM.

2. Place the codes 111111 and 11111 on the register of interrogation of the block of register RA [BR1] and the register of interrogation of the block of register RA [BR2] respectively at the same time.

2.1 Checking of these codes on concurrence of codes accordingly all 5 lines and all 6 columns of a code of the unknown letter is carried out and the result is recorded in appropriating categories of registers of fixing RS1 and RS2.

2.2 Received in RS1 and RS2, the pair of codes forms the first code of a sign of the given letter.

2.3 The received first code of a sign of the letter is compared to appropriating first codes of signs in memory of library. If the result of comparison is concurrent to one of codes of library, then it can be one of letters of M, Щ, Т, X, differently to pass to п.3.

3. Place the code $111 \mathrm{mmm}$ on registers of interrogation of the block of register RA [BR1] and the code mm101 on registers of interrogation of the block of register RA [BR2], where $\mathrm{m}$ is the disguised which is not participating in check on concurrence of codes.

3.1 Checking of these codes on concurrence of codes accordingly all 5 lines and all 6 columns of a code of the unknown letter is carried out and the result is recorded in appropriating categories of registers of fixing RS1 and RS2.
3.2 Received in RS1 and RS2, the second pair code forms the second code of a sign of the given letter.

3.3 The received second code of a sign of the letter is compared to appropriating second codes of signs in memory of library. If the result of comparison is concurrent to one of codes of library, the appropriating letter is distinguished. An opposite case of such symbol in memory of library is not present, that is, the symbol is not distinguished.

Work on algorithm for recognition of letters is explained on specific examples. On Figure 5, the concept of a code of letters of ARAM (in the center) and conditions of registers of interrogation and fixing is shown. Received in RS1 and RS2, the 11-digits code acts on RA [BR1] matrixes of library which result is compared to the codes of the first part of a matrix that is concurrent to one of the codes (shown in Figure 6). Work on this way, the algorithm for recognition of letters comes to an end.

On Figure 7-10, the consequences (performance of items) of algorithm for recognition of letter A are shown respectively. On Figure 7, the concept of a code of the letter ' $A$ ' in memory ARAM and conditions of register interrogation and fixing is shown. On Figure 8, the comparison result of a code of sign of the letter with codes of the first part of library: the code of the distinguished letter has coincided with codes of the letter A and $\mathrm{B}$, therefore the algorithm passes to item 3 according to which the second code of a sign of the letter (condition RA [BR1] and RA [BR2] is shown in Figure 9), which is compared to codes of the second part of library (shown in Figure 10). Thus, the result of comparison is concurrent to codes of signs of the letter 'A' [4]. 


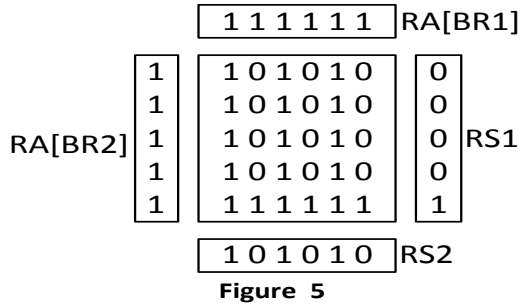

\begin{tabular}{lllll|}
1 & 1 & 1 & 1 & 1 \\
\hline
\end{tabular}

\begin{tabular}{|c|c|}
\hline 1 & 111000 \\
\hline 1 & 101000 \\
\hline 1 & 111000 \\
\hline 1 & 101000 \\
\hline 1 & 101000 \\
\hline
\end{tabular}

101000

Figure 7

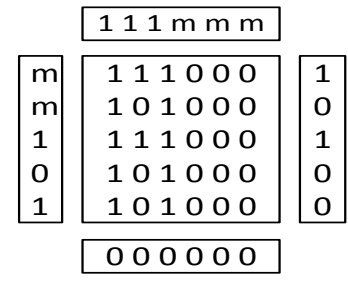

Figure 9

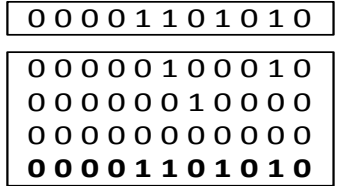

Figure 6

Figure 8

\begin{tabular}{|c|}
\hline 1010000 \\
\hline 10100000000 \\
\hline 10101010000 \\
\hline 10101010000 \\
\hline 10000000000 \\
\hline 00001000000 \\
\hline 10101011000 \\
\hline 00000000000 \\
\hline
\end{tabular}

Figure 10

Figure 5: The concept of a code of letters of ARAM (in the center) and conditions of registers of interrogation and fixing, Figure 6: Codes for comparable and Figure 7 to 10: The consequences (performance of items) of algorithm for recognition of letter $A$ are shown respectively.

\section{COMPARISON RESULT OF \\ SIMULATION IN ARAM AND RAM}

The following table shows a comparison chart of quantity of hits in ARAM and RAM for each Russian alphabet.

Table 1: Russian Alphabets with corresponding quantity of hits in ARAM and RAM

\begin{tabular}{|c|c|c|c|c|c|c|c|c|c|c|}
\hline X (Russian Alphabets) & A & $\mathbf{5}$ & B & $\boldsymbol{\Gamma}$ & д & $\mathbf{E}$ & ж & 3 & и & $\mathbf{K}$ \\
\hline$Y$ (Quantity of hits in ARAM) & 14 & 16 & 14 & 16 & 12 & 12 & 12 & 16 & 18 & 14 \\
\hline$Y$ (Quantity of hits in RAM) & 153 & 164 & 157 & 169 & 132 & 124 & 134 & 164 & 184 & 169 \\
\hline X (Russian Alphabets) & л & $\mathbf{M}$ & $\mathbf{H}$ & $\mathbf{0}$ & $\Pi$ & $\mathbf{P}$ & C & $\mathbf{T}$ & y & $\Phi$ \\
\hline$Y$ (Quantity of hits in ARAM) & 16 & 18 & 14 & 14 & 12 & 12 & 12 & 16 & 16 & 8 \\
\hline$Y$ (Quantity of hits in RAM) & 177 & 184 & 159 & 158 & 130 & 130 & 126 & 169 & 167 & 92 \\
\hline X (Russian Alphabets) & $\mathbf{x}$ & ц & 4 & ш & щ & b & $\mathbf{b}$ & э & Ю & я \\
\hline$Y$ (Quantity of hits in ARAM) & 14 & 14 & 14 & 14 & 10 & 8 & 16 & 14 & 14 & 14 \\
\hline Y (Quantity of hits in RAM) & 169 & 164 & 159 & 164 & 126 & 91 & 167 & 158 & 159 & 163 \\
\hline
\end{tabular}

In the following bar diagram, the $\mathrm{X}$-axis shows the Russian alphabets being compared and the $\mathrm{Y}$-axis represents the quantity (discrete value) of hits in ARAM and RAM (Classical Memory). 


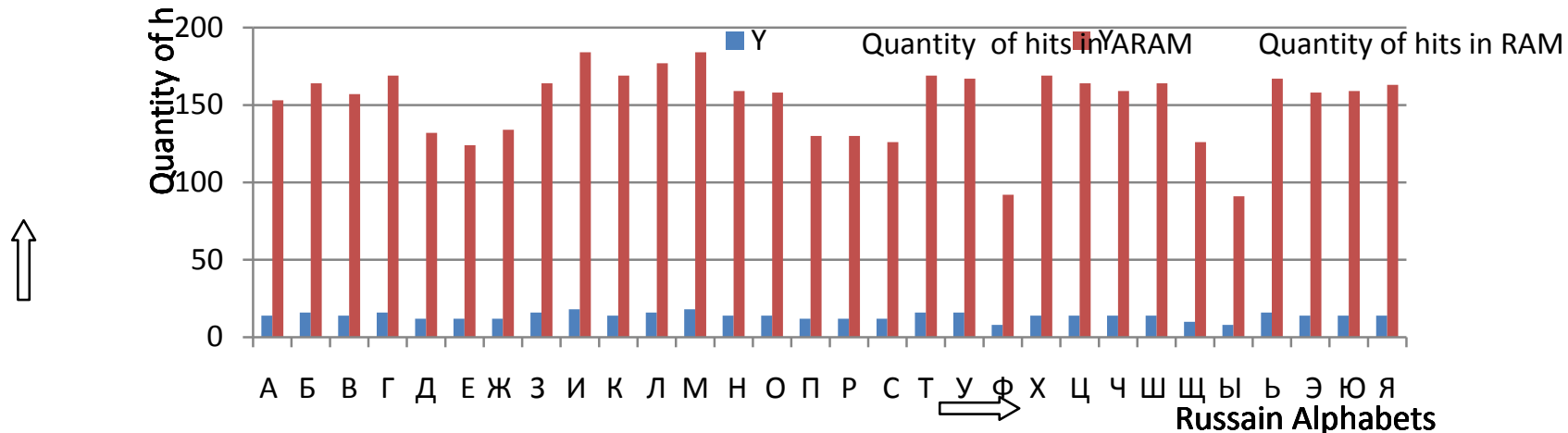

Figure 11: Bar diagram of comparison between ARAM and RAM

It has seen that from both Table 1 and Figure 11, the quantity of hits for every Russian alphabet in RAM is ten times more than the quantity of hits for same Russian alphabets in ARAM. For instance, the quantity of hits in RAM for the alphabet ' $\mathbf{E}$ ' is 164 whereas the quantity of hits in ARAM for the same alphabet is only 16 and so on.

\section{CONCLUSION}

In this paper, the goal was how to recognize a symbol or an alphabet in the associative cells of the ARAM and how many times an alphabet hits in ARAM and RAM. Here the letters of Russian Alphabets have been considered as instances.

From the comparison's result it can be deduced that, it is very easy to recognize the letters of the Russian Alphabets in the ARAM. Also these Russian alphabets can be easily processed i.e., the quantity of hits in RAM for an alphabet is greater than the quantity of hits in ARAM for the same alphabet. In other words, it can say that the required time to process an alphabet in an associative memory is relatively low. As a result an associative memory is very useful when considering high speed and it provides us to do work in an environment with more data within very short time.

In future, they will work on how to recognize threedimensional or solid objects in ARAM and compare the performance of the recognition of three-dimensional or solid objects between the RAM and the ARAM. That is, they will see if the ARAM is faster than the RAM to recognize the three-dimensional objects. They have already started their work and they will continue till the ultimate result.

\section{REFERENCES}

[1]. Ognev I. V., Boriss V. V. Intellectual systems of associative memory. M.: Radio and Svyaz, 1996.-176c.
[2]. Ognev I. V., Md. Abdul Malek, processing of matrixes in the associative environment. International forum of information-2000: Reports of the international conference «Information means and technologies ». 1719 октобря 2000г. In 3 vol. T.2.-M: publishing house "Stankin ", 2000г...-245.

[3]. Ognev I. V., Boriss V. V. Associative environment. M.: Radio and Svyaz, 2000.-312.

[4]. Dr. Md. Abdul Malek, Md. Mohibullah, Department of Computer Science and Engineering, Comilla University, Comilla, Bangladesh. Efficiency and Effectiveness of Associative Random Access Memory in Solving Difficult Tasks. BES Journal Vol 14 Issue 1-2, June2014.

\section{AUTHORS PROFILE}

Dr. Md. Abdul Malek who obtained his B.Sc. (Honors), M.Sc. and Ph.D. degree in Computer Science and Engineering from Moscow, Russia. At present, he is employed as an Assistant Professor in the department of Computer Science and Engineering (CSE) at Comilla University, Comilla, Bangladesh. He worked as an Assistant Professor at Moscow Power Engineering University in Moscow, Russia and University of Information Technology and Sciences in Dhaka, Bangladesh. His research activities involve Processing Data in Associative Random Access Memory (ARAM).

Md. Mohibullah who obtained B.Sc. (Engg.) in Computer Science and Engineering Department at Comilla University, Comilla, Bangladesh. He is now a student of M.Sc. (Thesis) at this university and a member (student) of Bangladesh Computer Society (BCS). His research interest includes Associative Random Access Memory (ARAM), Artificial Intelligent and Robotics . 